\title{
The light composite Higgs boson in strong extended technicolor
}

\author{
Kenneth Lane and Lukas Pritchett \\ Department of Physics, Boston University, \\ 590 Commonwealth Avenue, Boston, Massachusetts 02215, U.S.A. \\ E-mail: lane@physics.bu.edu, lpritch@bu.edu
}

ABSTRACT: This paper extends an earlier one describing the Higgs boson $H$ as a light composite scalar in a strong extended technicolor model of electroweak symmetry breaking. The Higgs mass $M_{H}$ is made much smaller than $\Lambda_{\text {ETC }}$ by tuning the ETC coupling very close to the critical value for electroweak symmetry breaking. The technicolor interaction, neglected in the earlier paper, is considered here. Its weakness relative to extended technicolor is essential to understanding the lightness of $H$ compared to the low-lying spin-one technihadrons. Technicolor cannot be completely ignored, but implementing technigluon exchange together with strong extended technicolor appears difficult. We propose a solution that turns out to leave the results of the earlier paper essentially unchanged. An argument is then presented that masses of the spin-one technifermion bound states, $\rho_{H}$ and $a_{H}$, are much larger than $M_{H}$ and, plausibly, controlled by technicolor. Assuming $M_{\rho_{H}}$ and $M_{a_{H}}$ are in the TeV-energy region, we identify $\rho_{H}$ and $a_{H}$ with the diboson excesses observed near $2 \mathrm{TeV}$ by ATLAS and CMS in LHC Run 1 data, and we discuss their phenomenology for Runs 2 and 3.

Keywords: Higgs Physics, Technicolor and Composite Models

ArXIV EPRINT: 1604.07085 


\section{Contents}

1 Overview 1

2 Adding TC to strong ETC $\quad 7$

3 Masses of the spin-one technihadrons $\quad 10$

4 Phenomenology of $\rho_{H}$ and $a_{H} \quad 12$

5 Summary and plans $\quad 16$

\section{Overview}

The discovery at CERN in 2012 of a Higgs boson, $H$, at $125 \mathrm{GeV}[1,2]$ — consistent so far with the lone Higgs boson of the standard model - has made untenable the original idea of technicolor as the source of electroweak symmetry breaking (EWSB) [3, 4]. In technicolor (TC), the new boson $H$ is interpreted as the lightest $0^{+}$bound state of technifermions. But, then, one expects other bound technihadrons as light as this scalar, especially the spin-one vector and axial vector states, and resonances this light have not been seen. ${ }^{1,2}$

An attractive dynamical alternative to $\mathrm{TC}$ is that $H$ is a composite state bound by strong interactions active well above the weak scale $\Lambda_{\mathrm{EW}}$ of several $100 \mathrm{GeV}$. But, unlike TC, all composite models and, indeed, all models so far of $H$ require some degree of finetuning [11-14] to be consistent with ATLAS and CMS measurements [15] and searches. ${ }^{3}$ This tuning may be loosely characterized by $\Lambda_{\mathrm{EW}}^{2} / \Lambda^{2}$, where $\Lambda$ is the physical scale of the new dynamics or particles stabilizing $M_{H}$.

Motivated by these considerations, we proposed a new composite model of $H$ employing strong extended technicolor (ETC) as the main driver of EWSB [16]. As in the standard model (SM) and TC, the fermions in this model transform as left-handed doublets and

\footnotetext{
${ }^{1}$ Refs. [5, 6] have argued that the mass of the lightest scalar technihadron is greatly reduced from its expected value of 100 s of $\mathrm{GeV}$ by the negative top-loop contribution. This requires a large top mass from extended technicolor and a large coupling of $\bar{t} t$ to the scalar, neither of which are explained in these references. These papers also ignore the strong-TC coupling of the scalar to the Goldstone bosons of EWSB. A simple model calculation shows that this positive loop-contribution overwhelms the negative one from top.

${ }^{2}$ Many papers have suggested that the light Higgs boson is a techni-dilaton, i.e., a pseudo-Goldstone boson (PGB) of spontaneously broken conformal symmetry in walking technicolor. See refs. [7-10] for a sampling. This is an appealing idea, but it is difficult to understand how such a light PGB can arise when the explicit breaking giving rise to its mass, namely spontaneous chiral symmetry breaking in technicolor, is as strong as the near-conformal dynamics of technicolor itself.

${ }^{3}$ https://twiki.cern.ch/twiki/bin/view/AtlasPublic/ExoticsPublicResults, https://twiki.cern.ch/twiki/bin/view/AtlasPublic/SupersymmetryPublicResults, https://twiki.cern.ch/twiki/bin/view/CMSPublic/PhysicsResultsEXO, https://twiki.cern.ch/twiki/bin/view/CMSPublic/PhysicsResultsSUS, https://twiki.cern.ch/twiki/bin/view/CMSPublic/PhysicsResultsB2G.
} 
right-handed singlets under $(\mathrm{SU}(2) \otimes \mathrm{U}(1))_{\mathrm{EW}}$. If the ETC interaction's strength exceeds a critical value, it generates nonzero top quark and technifermion masses, $m_{t}$ and $m_{T}$, thus breaking electroweak (EW) symmetry. Generically, these masses are of order the ETC scale, $\Lambda=\Lambda_{\mathrm{ETC}}$, of several $100 \mathrm{TeV}$. But this can be avoided if the ETC coupling is tuned to within $\mathcal{O}\left(m_{t}^{2} / \Lambda^{2}\right)$ of its critical value [17]. It was shown in ref. [18] that ETC then generates a composite complex EW doublet consisting of a scalar $H$ with vacuum expectation value (vev) $v=\mathcal{O}\left(m_{t}\right)$ plus three Goldstone bosons, the longitudinal components of $W$ and $Z$. The scalar has a large Yukawa coupling $\Gamma_{t} \simeq m_{t} / v$ to the top quark and a mass $M_{H}=\mathcal{O}\left(m_{t}\right) \ll \Lambda$. Such a model might account for the Higgs boson's exceptional lightness.

In ref. [16] we presented an explicit realization of such a model. The model is a generalization of the topcolor model of Bardeen, Hill and Lindner (BHL) [19], and followed that paper's demonstration of the massless poles in fermion-antifermion scattering channels that couple to the weak currents and of the massive pole in the $0^{+}$channel. An important difference with refs. [17-19] is that three four-fermion interactions, not just one, are required and there is a special relation among their coupling strengths. The details are summarized below. Another point of departure of the new model is that it pointed out the likely presence of high-mass, $\rho$-like diboson resonances as an important, perhaps the most important, experimental consequence of our model (see sections 3 and 4 ).

This model's approach differs from the popular view that the Higgs is a pseudoGoldstone boson; see, e.g., refs. [11, 13, 20]. Not only is our model's $H$ not a PGB, but there are no partners of the top quark and weak bosons to cancel their quadratically divergent contributions to its mass. Rather, this quadratic divergence is removed by the condition that $m_{t}$ and $m_{T}$ are much less than $\Lambda$. This is a significant fine-tuning but, as explained below eq. (1.7), this is the only one in the model. Thus, there is no need to fine-tune partners' masses and couplings to explain why they haven't been seen in LHC experiments.

In ref. [16] TC dynamics were not included. But TC cannot be ignored. First, there must be an unbroken TC subgroup of ETC. If all its symmetries were spontaneously broken, ETC would be infrared free at energies below the ETC boson masses. It is unclear whether such a theory can be free in the ultraviolet [21]. Second, at the scale $\Lambda_{\mathrm{TC}} \lesssim 1 \mathrm{TeV} \ll \Lambda_{\mathrm{ETC}}=\Lambda$, the TC gauge coupling $\alpha_{\mathrm{TC}}$ becomes strong enough that it can break EW symmetry all by itself. So, this is a situation with two very different but nonetheless important energy scales. ETC is the dominant force in driving EWSB and making the Higgs boson light. But what sets the mass scale for the technihadrons, the bound states of technifermions? Are they bound by TC alone or, like the Higgs boson, by ETC, or by some cooperative combination? A major of purpose of this paper is to include the effects of TC on EWSB and to estimate the mass scale of the spin-one technihadrons. Because of their potential experimental importance, we need to know whether they are much heavier than $H$ and, if so, whether they are within reach of the LHC experiments.

That TC must play a minor role compared to ETC in EW symmetry breaking was not emphasized in ref. [16], even though it was one of the two main approximations of that paper. The relative contributions that TC and ETC make in binding the spin-one technihadrons and generating their masses, and the requirement that the Higgs boson is much lighter than they, is what brings this issue to the fore. 
We now review the main results of ref. [16]: the fermions and their ETC interaction, the fine-tuned gap equations for the fermions' masses, the Higgs mass, and the principal results of EWSB. Then we preview the rest of this paper.

The model of ref. [16] involved the third-generation quarks and a single doublet of technifermions transforming under $(\mathrm{SU}(2) \otimes \mathrm{U}(1))_{\mathrm{EW}}$, ordinary color $\mathrm{SU}(3)_{C}$ and technicolor $\mathrm{SU}\left(N_{\mathrm{TC}}\right)$ as follows:

$$
\begin{aligned}
& q_{L}=\left(\begin{array}{l}
t \\
b
\end{array}\right)_{L} \in\left(\mathbf{2}, \frac{1}{6}, \mathbf{3}, \mathbf{1}\right), \quad t_{R} \in\left(\mathbf{1}, \frac{2}{3}, \mathbf{3}, \mathbf{1}\right), \quad b_{R} \in\left(\mathbf{1},-\frac{1}{3}, \mathbf{3}, \mathbf{1}\right), \\
& T_{L}=\left(\begin{array}{c}
U \\
D
\end{array}\right)_{L} \in\left(\mathbf{2}, 0, \mathbf{1}, \boldsymbol{d}_{\mathrm{TC}}\right), \quad U_{R} \in\left(\mathbf{1}, \frac{1}{2}, \mathbf{1}, \boldsymbol{d}_{\mathrm{TC}}\right), \quad D_{R} \in\left(\mathbf{1},-\frac{1}{2}, \mathbf{1}, \boldsymbol{d}_{\mathrm{TC}}\right) .
\end{aligned}
$$

Here, $\boldsymbol{d}_{\mathrm{TC}}$ denotes the $d_{\mathrm{TC}}$-dimensional TC representation of the technifermions, not necessarily the fundamental representation of dimension $N_{\mathrm{TC}}$. Light quarks and leptons and other technifermions were not dealt with, but they may be included, e.g., as outlined in ref. [19].

The ETC interaction inducing EWSB at energies below $\Lambda$ was taken to be the straightforward generalization for these fermions of the $\mathrm{SU}(2)_{L} \otimes \mathrm{U}(1)_{R}$-invariant model of ref. [19]:

$$
\mathcal{L}_{\mathrm{ETC}}=G_{1} \bar{q}_{L}^{i a} t_{R a} \bar{t}_{R}^{b} q_{L i b}+G_{2}\left(\bar{q}_{L}^{i a} t_{R a} \bar{U}_{R}^{\alpha} T_{L i \alpha}+\text { h.c. }\right)+G_{3} \bar{T}_{L}^{i \alpha} U_{R \alpha} \bar{U}_{R}^{\beta} T_{L i \beta} .
$$

The $\mathrm{SU}(2)_{\mathrm{EW}}$ and color-SU $(3)_{C}$ and $\mathrm{SU}\left(N_{\mathrm{TC}}\right)$ indices, $i$ and $a, b$ and $\alpha, \beta$ are summed over. This interaction is obtained by Fierzing ETC contact terms of left times righthanded currents. The color and TC indices appearing here do not correspond to exchange of massless color and TC gluons. The couplings $G_{1,2,3}$ are positive and of $\mathcal{O}\left(1 / \Lambda^{2}\right) .{ }^{4}$

In the neglect of EW interactions, the model has an $\left(\mathrm{SU}(2)_{L} \otimes \mathrm{U}(1)_{R}\right)_{q} \otimes\left(\mathrm{SU}(2)_{L} \otimes\right.$ $\left.\mathrm{U}(1)_{R}\right)_{T}$ flavor symmetry that is explicitly broken to $\mathrm{SU}(2)_{L} \otimes \mathrm{U}(1)$ by the $G_{2}$-term. If $\mathcal{L}_{\text {ETC }}$ generates both $t$ and $U$ masses and $G_{2} \neq 0$, this flavor symmetry is spontaneously broken to U(1) and just three Goldstone bosons appear. In fact, $G_{2}$ must not equal zero; if it were, this would be a two-Higgs doublet model with an extra triplet of Goldstone bosons. They would acquire only very small EW masses [22] and, so, are excluded experimentally. With $G_{2} \neq 0$, this model has exactly one Higgs boson. Its vev is $v=246 \mathrm{GeV}$, setting the scale for $m_{t, U}$; see eqs. (1.10), (1.12) below. The low-energy theory below the ETC scale $\Lambda$ is the standard model with spontaneously broken $\mathrm{SU}(2) \otimes \mathrm{U}(1)$ and massive EW gauge bosons, a dynamical Higgs boson and its couplings to $t$ and $U$, and technihadrons. Below the technihadron masses, $M_{\mathrm{TC}}$, their effects on SM Higgs couplings are suppressed by $1 / M_{\mathrm{TC}}^{2}$, in accord with all measurements so far. ${ }^{5}$

\footnotetext{
${ }^{4}$ The $D$-technifermion gets no hard mass from ETC in this model. It is not difficult to add terms that generate $m_{D} \neq 0$, but not so easy to maintain both $m_{D} \cong m_{U}$ and $m_{b} \ll m_{t}$ at scale $\Lambda$. It was pointed out in ref. [18] that the renormalization group equations for the $U$ and $D$ Yukawa couplings have an infrared fixed point that tends to equalize $m_{U}$ and $m_{D}$ at $\Lambda_{\mathrm{TC}}$.

${ }^{5}$ This was emphasized in ref. [19] in which the authors presented the effective Lagrangian with the Higgs boson, and discussed its renormalization.
} 
The TC interaction was neglected in ref. [16], and calculations were carried out in the Nambu-Jona-Lasinio (NJL) approximation of large $d_{\mathrm{TC}}$ and $N_{C}$. The gap equations for the hard masses $m_{t}$ and $m_{U}$, assumed much less than $\Lambda$ and renormalized at the scale $\Lambda$, are

$$
\begin{aligned}
m_{t} & =-\frac{1}{2} G_{1}\langle\bar{t} t\rangle-\frac{1}{2} G_{2}\langle\bar{U} U\rangle \\
& =\frac{G_{1} N_{C} m_{t}}{8 \pi^{2}}\left(\Lambda^{2}-m_{t}^{2} \ln \frac{\Lambda^{2}}{m_{t}^{2}}\right)+\frac{G_{2} d_{\mathrm{TC}} m_{U}}{8 \pi^{2}}\left(\Lambda^{2}-m_{U}^{2} \ln \frac{\Lambda^{2}}{m_{U}^{2}}\right) ; \\
m_{U} & =-\frac{1}{2} G_{2}\langle\bar{t} t\rangle-\frac{1}{2} G_{3}\langle\bar{U} U\rangle \\
& =\frac{G_{2} N_{C} m_{t}}{8 \pi^{2}}\left(\Lambda^{2}-m_{t}^{2} \ln \frac{\Lambda^{2}}{m_{t}^{2}}\right)+\frac{G_{3} d_{\mathrm{TC}} m_{U}}{8 \pi^{2}}\left(\Lambda^{2}-m_{U}^{2} \ln \frac{\Lambda^{2}}{m_{U}^{2}}\right) .
\end{aligned}
$$

We can treat the dimensionalities $N_{C}$ and $d_{\text {TC }}$ as independent and even continuous parameters for which the gap equations hold. Then, multiplying eq. (1.3) by $m_{U}$ and eq. (1.4) by $m_{t}$, and varying $N_{C}$ and $d_{\text {TC }}$ independently, the two resulting equations can be true for nonzero $m_{t}$ and $m_{U}$ if and only if

$$
\frac{G_{1} N_{C} m_{U}}{8 \pi^{2}}=\frac{G_{2} N_{C} m_{t}}{8 \pi^{2}} \text { and } \frac{G_{2} d_{\mathrm{TC}} m_{U}}{8 \pi^{2}}=\frac{G_{3} d_{\mathrm{TC}} m_{t}}{8 \pi^{2}} .
$$

Hence,

$$
G_{2}=G_{1} \frac{m_{U}}{m_{t}}=G_{3} \frac{m_{t}}{m_{U}} .
$$

Then, eqs. (1.3)-(1.6) yield the condition:

$$
\begin{aligned}
& \frac{G_{1} N_{C}}{8 \pi^{2}}\left(\Lambda^{2}-m_{t}^{2} \ln \frac{\Lambda^{2}}{m_{t}^{2}}\right)+\frac{G_{3} d_{\mathrm{TC}}}{8 \pi^{2}}\left(\Lambda^{2}-m_{U}^{2} \ln \frac{\Lambda^{2}}{m_{U}^{2}}\right) \\
& \quad=G_{2}\left[\frac{N_{C} m_{t}}{8 \pi^{2} m_{U}}\left(\Lambda^{2}-m_{t}^{2} \ln \frac{\Lambda^{2}}{m_{t}^{2}}\right)+\frac{d_{\mathrm{TC}} m_{U}}{8 \pi^{2} m_{t}}\left(\Lambda^{2}-m_{U}^{2} \ln \frac{\Lambda^{2}}{m_{U}^{2}}\right)\right]=1 .
\end{aligned}
$$

It was shown in ref. [16] that $m_{t}$ and $m_{U}$ are comparable and, so, the three $G_{i}$ are comparable as well.

Eq. (1.7) is the expression of strong ETC in our model. This raises the question of whether TC, the unbroken subgroup of ETC, must be strongly coupled at the ETC scale. If it is, that would contradict the thesis of this paper that TC is and must be a weak perturbation on ETC insofar as triggering EWSB is concerned. But the $G_{i}$ are in fact independent of the ETC gauge coupling, $g_{\mathrm{ETC}}$. Just as in the standard weak interaction at low energies, they are essentially equal to $g_{\mathrm{ETC}}^{2} / M_{\mathrm{ETC}}^{2}$, where $M_{\mathrm{ETC}} \propto g_{\mathrm{ETC}}$ times a Goldstone boson decay constant. Thus, the gauge coupling may be relatively weak while the four-fermion couplings are strong in the sense of eq. (1.7).

Requiring $m_{t}, m_{U} \ll \Lambda$ is this model's only fine tuning. Once eq. (1.7) is enforced in the fermion-antifermion scattering amplitudes in the spin-zero channels, all other sensitivity to the cutoff $\Lambda$ is logarithmic. The mass parameters $m_{t}, m_{U}, M_{W}, M_{H}$ and $\Lambda$ are not independent. In the large- $N$ approximation, their magnitude is set by requiring eq. (1.12) below, and the Higgs mass $M_{H}$ is then determined by $m_{t}, m_{U}$ and $N_{C}, d_{\mathrm{TC}}$. 
The fermion-antifermion scattering amplitudes (involving $t$ and/or $U$ ) have a pole in the $0^{+}$channel at squared c.m. energy $p^{2}=M_{H}^{2}$, where $M_{H}$ is the solution of

$$
\begin{aligned}
& N_{C} m_{t}^{2}\left(M_{H}^{2}-4 m_{t}^{2}\right) \int_{0}^{1} d x \ln \left(\frac{\Lambda^{2}}{m_{t}^{2}-M_{H}^{2} x(1-x)}\right) \\
& +d_{\mathrm{TC}} m_{U}^{2}\left(M_{H}^{2}-4 m_{U}^{2}\right) \int_{0}^{1} d x \ln \left(\frac{\Lambda^{2}}{m_{U}^{2}-M_{H}^{2} x(1-x)}\right)=0 .
\end{aligned}
$$

This $M_{H}$ is the Higgs boson mass at scale $\Lambda$. A good approximation to the solution of eq. (1.8) is

$$
M_{H}=2 \sqrt{\frac{N_{C} m_{t}^{4}+d_{\mathrm{TC}} m_{U}^{4}}{N_{C} m_{t}^{2}+d_{\mathrm{TC}} m_{U}^{2}}}
$$

Thus, $M_{H}$ is indeed of order $m_{t}, m_{U}$ and all these masses are much less than $\Lambda$ because the ETC couplings have been tuned to be very close to the critical point at which EWSB first occurs.

The fermion-antifermion scattering amplitudes in the charged and neutral pseudoscalar channels have Goldstone poles at $p^{2}=0$. These poles appear in the $W$ and $Z$ propagators, $g_{2}^{-2} D_{W}(p)$ and $\left(g_{1}^{2}+g_{2}^{2}\right)^{-1} D_{Z}(p)$, with residues

$$
f_{W}^{2}\left(p^{2}\right)=\frac{1}{16 \pi^{2}} \int_{0}^{1} d x x\left[N_{C} m_{t}^{2} \ln \left(\frac{\Lambda^{2}}{m_{t}^{2} x-p^{2} x(1-x)}\right)+d_{\mathrm{TC}} m_{U}^{2} \ln \left(\frac{\Lambda^{2}}{m_{U}^{2} x-p^{2} x(1-x)}\right)\right]
$$

and

$$
\begin{aligned}
f_{Z}^{2}\left(p^{2}\right)= & \frac{1}{32 \pi^{2}} \int_{0}^{1} d x\left[N_{C} m_{t}^{2} \ln \left(\frac{\Lambda^{2}}{m_{t}^{2}-p^{2} x(1-x)}\right)+d_{\mathrm{TC}} m_{U}^{2} \ln \left(\frac{\Lambda^{2}}{m_{U}^{2}-p^{2} x(1-x)}\right)\right] \\
& +\frac{N_{C} p^{2}}{16 \pi^{2}} \int_{0}^{1} d x \frac{1}{3} x(1-x) \ln \left(\frac{-p^{2} x(1-x)}{m_{t}^{2}-p^{2} x(1-x)}\right) .
\end{aligned}
$$

The EW mass scale is introduced by setting

$$
f_{W}^{2}(0)=1 /\left(4 \sqrt{2} G_{F}\right)=(123 \mathrm{GeV})^{2} \cong M_{W}^{2} / g_{2 W}^{2}(0) .
$$

The $\rho$-parameter,

$$
\rho \cong \frac{f_{W}^{2}(0)}{f_{Z}^{2}(0)}=\frac{\left[N_{C} m_{t}^{2}\left(\ln \left(\Lambda^{2} / m_{t}^{2}\right)+\frac{1}{2}\right)+d_{\mathrm{TC}} m_{U}^{2}\left(\ln \left(\Lambda^{2} / m_{U}^{2}\right)+\frac{1}{2}\right)\right]}{\left[N_{C} m_{t}^{2} \ln \left(\Lambda^{2} / m_{t}^{2}\right)+d_{\mathrm{TC}} m_{U}^{2} \ln \left(\Lambda^{2} / m_{U}^{2}\right)\right]},
$$

is just a few percent greater than one. This is spurious. The deviation of $\rho$ from unity in eq. (1.13) is due to the factors of $1 / 2$ in the numerator. Those factors should not have been included in ref. [16] because the calculations of $f_{W}^{2}(0)$ and $f_{Z}^{2}(0)$ where done in the leading-log approximation (in the cutoff/ETC scale $\Lambda$ ). Corrections to this approximation are unknown.

Table 1 contains numerical results for the model obtained from a simple scheme described in ref. [16].

In section 2 we discuss the difficulty of adding an interaction involving dynamical TCgluon exchange to the ETC contact interaction in eq. (1.2) and propose an approximation 


\begin{tabular}{|c|c|c|c|c|c|}
\hline$\Lambda$ & $m_{t}$ & $m_{U}$ & $M_{H}$ & $\Gamma_{t}$ & $v=\sqrt{2} m_{t} / \Gamma_{t}$ \\
\hline $20 \mathrm{TeV}$ & $134 \mathrm{GeV}$ & $167 \mathrm{GeV}$ & $330 \mathrm{GeV}$ & 0.783 & $242 \mathrm{GeV}$ \\
$500 \mathrm{TeV}$ & $118 \mathrm{GeV}$ & $126 \mathrm{GeV}$ & $250 \mathrm{GeV}$ & 0.685 & $244 \mathrm{GeV}$ \\
\hline$\Lambda$ & $\rho$ & $g_{1}$ & $g_{2}$ & $M_{W}$ (pole) & $M_{Z}$ (pole) \\
\hline $20 \mathrm{TeV}$ & 1.0520 & 0.3941 & 0.7187 & 80.8 & 91.0 \\
$500 \mathrm{TeV}$ & 1.0301 & 0.4230 & 0.7714 & 80.6 & 93.0 \\
\hline
\end{tabular}

Table 1. The fermion masses, Higgs boson mass, $\rho$-parameter, $\mathrm{SU}(2) \otimes \mathrm{U}(1))_{\mathrm{EW}}$ couplings and the $W, Z$-pole masses calculated for ETC scales $\Lambda=20$ and $500 \mathrm{TeV}$. The top mass is an input determined by renormalizing from its value of $173 \mathrm{GeV}$. The Higgs boson's vev $v=\sqrt{2} m_{t} / \Gamma_{t}$ is determined as a check on the calculation of the $\bar{t} t$ scattering amplitude in the scalar channel, where $\Gamma_{t}^{2}(\Lambda) / 2$ is the residue of the Higgs pole. The calculation scheme used is described in ref. [16]. As noted in the text, the deviations of $\rho$ from one are not reliably calculated.

that surmounts the problem for fermion-antifermion scattering in the spin-zero channel. The approximation is inspired by analyses of the effect of TC on the Schwinger-Dyson equation for the technifermion dynamical mass function, $\Sigma(p)$ [23, 24]. In section 3 we take up the matter of estimating the masses of the lightest spin-one vector and axial vector bound states, analogs of $\rho, \omega$ and $a_{1}$. We shall refer to them as $\rho_{H}, \omega_{H}$ and $a_{H}$ to emphasize their relation to the composite Higgs boson $H$. In this strong-ETC model, it is not obvious a priori whether their masses are of order $\Lambda_{\mathrm{ETC}}, \Lambda_{\mathrm{TC}}$ or something else, though that is a question of obvious phenomenological importance. We present a calculation that suggests they are of $\mathcal{O}\left(\Lambda_{\mathrm{TC}}\right)$. As in any strong interaction theory, a more precise estimate is technically difficult. Assuming they are within reach of LHC Runs $2+3$, their LHC phenomenology is discussed in section $4 .{ }^{6}$ There we review our recent proposal [25] that $\rho_{H}$ and $a_{H}$ are the source of the apparent diboson $(V V$ and $V H$, where $V=W, Z$ ) resonances near $2 \mathrm{TeV}$ observed by ATLAS and CMS in their Run 1 data [26-31] and we propose refined tests of our hypothesis for Runs $2+3$. New limits on diboson resonances from Run 2 data of $36 \mathrm{fb}^{-1}$ are also discussed in section 4 .

There has been much previous work using the NJL mechanism $[32,33]$ to describe the Higgs boson, including especially refs. [19, 34-36]. Topcolor led to the top-seesaw models of Dobrescu and Hill [37] and Chivukula, et al. [38] and, more recently, refs. [39, 40]. ${ }^{7}$ Bar-Shalom and collaborators proposed a "hybrid model" with a dynamical Higgs-like scalar plus an elementary scalar to describe $H$ [41, 42]. They used an NJL Lagrangian with fourth generation quarks interacting via a topcolor interaction with scale $\Lambda \sim 1 \mathrm{TeV}$ to generate the dynamical scalar. Apart from the use of the NJL bubble approximation, these models do not resemble ours, and the use of fourth generation quarks is reminiscent of the top-seesaw mechanism. The top-seesaw models involve mixing the top quark with another quark which is a weak isosinglet. That is not what happens in our model. The technifermion $U$ carries technicolor, not ordinary color, and its left-handed component is

\footnotetext{
${ }^{6}$ A preliminary discussion appeared in ref. [16].

${ }^{7}$ The last two papers contain a large bibliography of related work.
} 


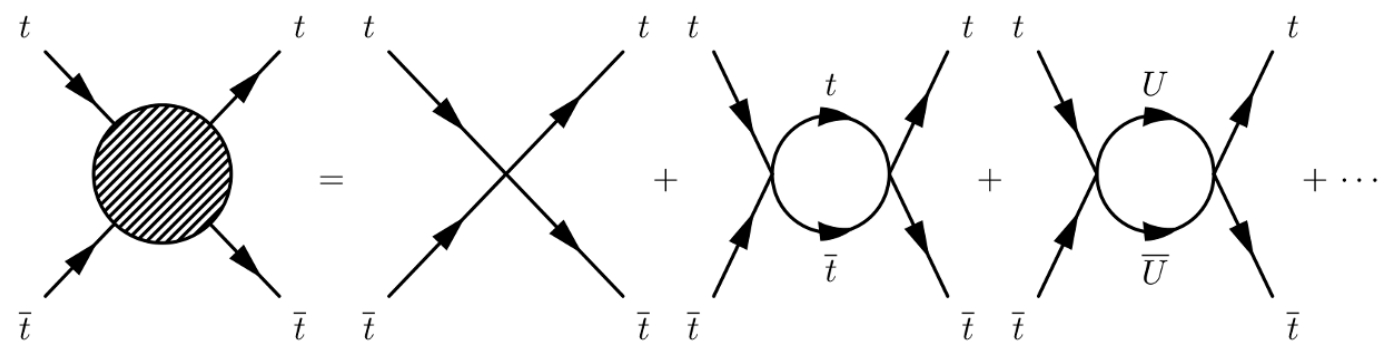

Figure 1. The $\bar{t} t$ scattering amplitude in the $J^{P}=0^{+}$channel with the four-fermion kernel given by the terms in eq. (2.1).

in a weak isodoublet, like the quarks and leptons. It does not mix with the top or any other quark.

Di Chiara, et al., proposed a model of $H$ based on TC and ETC [43, 44], using an ETC Lagrangian similar to eq. (1.2). Their model bears no further resemblance to ours. They assume that ETC plays no role in EWSB. But, through a sequence of calculations, they argue that ETC lowers their Higgs boson's mass from $\mathcal{O}(1 \mathrm{TeV})$ to $125 \mathrm{GeV}$. Finally, the authors of ref. [45] proposed an interesting variation on the Higgs boson as a PGB of the familiar $\mathrm{SO}(5) \rightarrow \mathrm{SO}(4)$ model. They used strong ETC-like contact interactions to drive this symmetry breakdown, and constructed a UV completion of this model.

\section{Adding TC to strong ETC}

In ref. [16], the Higgs and Goldstone bosons were seen as poles in the fermion-antifermion scattering amplitudes calculated in the large- $N$, weak-TC limit. Figure 1 shows the first few terms in $\bar{t} t \rightarrow \bar{t} t$ in the $J^{P}=0^{+}$channel. The four-fermion vertices are the appropriate terms in

$$
\mathcal{L}_{\mathrm{ETC}}=\frac{1}{4}\left[G_{1} \bar{t}^{a} t_{a} \bar{t}^{b} t_{b}+G_{2}\left(\bar{t}^{a} t_{a} \bar{U}^{\alpha} U_{\alpha}+\text { h.c. }\right)+G_{3} \bar{U}^{\alpha} U_{\alpha} \bar{U}^{\beta} U_{\beta}\right] .
$$

From eq. (1.6), $G_{2}^{2}=G_{1} G_{3}$, and this condition makes the scattering amplitudes geometric sums, with poles corresponding to the Higgs and three Goldstone bosons. The Higgs polemass condition eq. (1.8) follows once eq. (1.7) is imposed to eliminate the $\Lambda^{2}$-divergence in the $0^{+}$scattering amplitude.

In the large- $N$ approximation, the inclusion of TC-gluon exchange between the technifermions is accomplished by using the kernel $\mathcal{K}_{0^{+}}$in figure 2 . The TC-gluon term of this kernel is the familiar ladder approximation. The difficulty with it is how to deal with the momentum carried by the TC-gluon and, worse, whether the sum is a geometric series for which something like eq. (1.7) eliminates the $\Lambda^{2}$-divergence.

The only situation we know in which the ETC+TC kernel in figure 2 has been used successfully is in studies of the dynamical mass function $\Sigma\left(p^{2}\right)$ in the technifermion propagator $S^{-1}(p)=\not p A\left(p^{2}\right)-\Sigma\left(p^{2}\right)$ (where $A\left(p^{2}\right)=1$ in the Landau gauge ladder approximation) $[23,24]$. Remembering that the ETC boson mass $\Lambda$ is a physical cutoff of momentum integrals whose integrands are strongly damped above $\Lambda$, a good approximation to the 


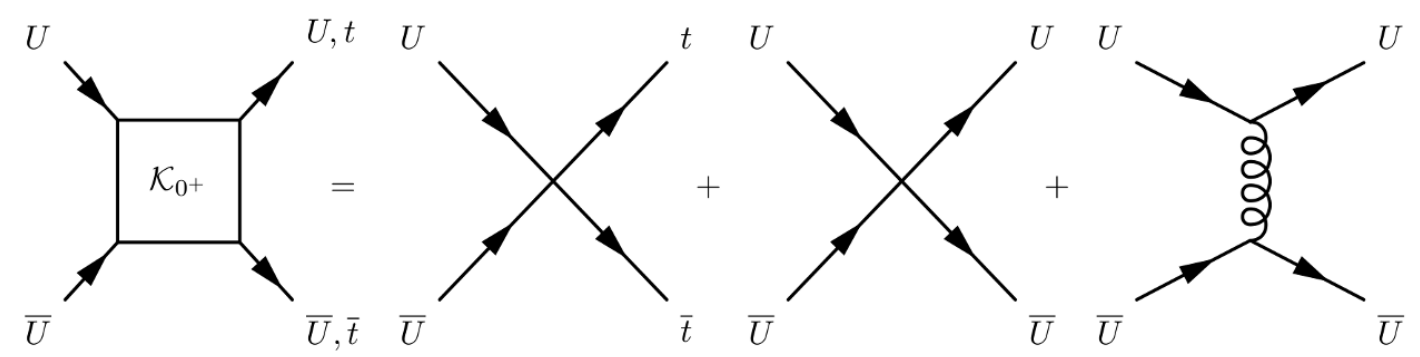

Figure 2. The kernel for scattering of $\bar{U} U \rightarrow \bar{t} t$ and $\bar{U} U \rightarrow \bar{U} U$ including ETC contact terms and one-TC-gluon exchange.

Schwinger-Dyson gap equation for $\Sigma\left(p^{2}\right)$ is (for zero bare mass and Euclidean momentum $p \lesssim \Lambda)$

$$
\Sigma\left(p^{2}\right)=\lambda \int_{0}^{\Lambda^{2}} d k^{2} \frac{k^{2}}{\Lambda^{2}} \frac{\Sigma\left(k^{2}\right)}{k^{2}+\Sigma^{2}\left(k^{2}\right)}+\frac{1}{4 \alpha_{c}} \int_{0}^{\Lambda^{2}} d k^{2} \alpha_{\mathrm{TC}}\left(M^{2}\right) \frac{k^{2}}{M^{2}} \frac{\Sigma\left(k^{2}\right)}{k^{2}+\Sigma^{2}\left(k^{2}\right)} .
$$

We consider a simplified model with just $G_{3}$ contributing to $\Sigma$. Then $\lambda=G_{3} d_{\mathrm{TC}} \Lambda^{2} / 8 \pi^{2}$; $\alpha_{\mathrm{TC}}$ is the running TC gauge coupling; $\alpha_{c}$ is the critical value of $\alpha_{\mathrm{TC}}$ for spontaneous chiral symmetry breaking in a pure-technicolor theory [46]; its value in the ladder approximation is $\pi / 3 C_{2}\left(\mathbf{d}_{\mathrm{TC}}\right)$; finally, $M^{2}=\max \left(k^{2}, p^{2}\right)$.

In a pure ETC theory, $\Sigma(0)=0$ for $\lambda<1$, there is a (presumed) second-order phase transition at $\lambda=1$, and $\Sigma(0)$ rises rapidly to $\mathcal{O}(\Lambda)$ just above the transition. In a pure asymptotically-free TC theory, $\alpha_{\mathrm{TC}}$ reaches $\alpha_{c}$ at a scale $\Lambda_{c}, \Sigma(0) \simeq \Sigma\left(\Lambda_{c}\right)=\mathcal{O}\left(\Lambda_{c}\right)$, and $\Sigma\left(p^{2}\right)$ falls off approximately as $\Lambda_{c}^{3} / p^{2}$ when $\alpha_{\mathrm{TC}}$ becomes weak [47]. Ref. [23] studied eq. (2.2) for constant $\alpha_{\mathrm{TC}}$. For $\alpha_{\mathrm{TC}}<\alpha_{c}$, the behavior of $\Sigma(0)$ was as in a pure-ETC theory except that, for $\alpha_{\mathrm{TC}}<\alpha_{c}$, the phase transition occurred at

$$
\lambda_{\alpha_{\mathrm{TC}}}=\left[\frac{1+\sqrt{1-\alpha_{\mathrm{TC}} / \alpha_{c}}}{2}\right]^{2} .
$$

Takeuchi studied the gap equation for a running $\alpha_{\mathrm{TC}}$ governed by the one-loop beta function $\beta\left(\alpha_{\mathrm{TC}}\right)=-b_{1} \alpha_{\mathrm{TC}}^{2}$, with $b_{1}>0$ [24]. So long as $\Lambda_{c} \ll \Lambda$ (as we expect), he found that $\Sigma(0)=\mathcal{O}\left(\Lambda_{c}\right)$ for $\lambda<\lambda_{\alpha_{\mathrm{TC}}}{ }^{8}$ Here, $\alpha_{\mathrm{TC}} \simeq \alpha_{\mathrm{TC}}(\Lambda)$. At this critical value of $\lambda$, there is a smooth but rapid transition up to $\Sigma(0)=\Lambda /$ few. The transition is more abrupt for small $\alpha_{\mathrm{TC}}(\Lambda) / \alpha_{c}$ so that $\lambda_{\alpha_{\mathrm{TC}}} \sim 1$. The reason that $\alpha_{\mathrm{TC}}(\Lambda)$ is the controlling coupling for $\lambda_{\alpha_{\mathrm{TC}}}$ is that, for this $\beta$-function and $\Lambda_{c} \ll \Lambda, \alpha_{\mathrm{TC}} \simeq \alpha_{\mathrm{TC}}(\Lambda) \ll \alpha_{c}$ and it is slowly running for most of the momentum range in the gap equation integral. We have verified Takeuchi's results for a more realistic walking-TC $\beta$-function, one with an infrared fixed point [48]. We also studied the momentum dependence of $\Sigma\left(p^{2}\right)$. For $\lambda<\lambda_{\alpha_{\mathrm{TC}}(\Lambda)}$, we found that $\Sigma$ is small and falls off approximately as $1 / p^{2}$ for $\Lambda_{c} \lesssim p \lesssim \Lambda$, as for a pure-TC dynamical mass. At the critical $\lambda, \Sigma(p)$ rises rapidly to $\mathcal{O}(\Lambda / 10)$ and then remains nearly constant in $p$, as for a hard mass.

\footnotetext{
${ }^{8}$ Takeuchi's definition of $\Lambda_{c}$ is lower than the scale at which $\alpha_{\mathrm{TC}}=\alpha_{c}$. This appears to be an artifact of his calculation procedure at low momenta.
} 
This is an important result for us. In the weak dynamical-TC case needed for a light composite Higgs with $M_{H}^{2} \ll M_{\rho_{H}}^{2}$, this behavior of $\Sigma$ is nearly what we get in the complete neglect of TC: it is much smaller than $\Lambda$ below $\lambda_{\alpha_{\mathrm{TC}}}$ and rises abruptly above, almost to $\mathcal{O}(\Lambda)$. ( $\operatorname{Had} \alpha_{\mathrm{TC}}(\Lambda)$ been large, the transition from small to large $\Sigma$ would have been gradual and there could be no large separation between $H$ and $\rho_{H}$ masses.) The critical $\lambda_{\alpha_{\mathrm{TC}}}$ is smaller than one because, to a good approximation, TC produces an interaction in the spin-zero channels of the same form and sign as the $G_{3}$-term in $\mathcal{L}_{\text {ETC. }}$ Thus, a smaller value of $G_{3}$, i.e., $\lambda$, is needed to trigger the phase transition. The critical value of the sum of the two interaction strengths is still fixed by a condition like eq. (1.7); i.e., the effective $G_{3}$ in $\mathcal{L}_{\text {ETC }}$ is essentially unchanged. Since $\mathcal{L}_{\text {ETC }}$ is the interaction determining the Higgs and Goldstone poles and their couplings to fermions in the large- $N$ limit, the results reviewed in section 1 are also unchanged.

To see this in detail, we use the fact that the TC coupling involved in the EW phase transition is approximately $\alpha_{\mathrm{TC}}(\Lambda)$. The relevant TC interaction then involves exchange of a technigluon with Euclidean momentum transfer $\approx-\Lambda^{2}$,

$$
\mathcal{L}_{\mathrm{TC}}=-\frac{3 \pi \alpha_{\mathrm{TC}}(\Lambda)}{2 \Lambda^{2}} \sum_{A} \bar{T} \gamma^{\mu} t_{A} T \bar{T} \gamma_{\mu} t_{A} T
$$

where $T=(U, D)$ is the technifermion doublet, $t_{A}$ are the TC generators in the representation $\boldsymbol{d}_{\mathrm{TC}}$, and other indices are suppressed. A factor of $3 / 4$ has been introduced into $\mathcal{L}_{\mathrm{TC}}$ to compensate for using Landau instead of Feynman gauge. ${ }^{9}$ Use

$$
\sum_{A}\left(t_{A}\right)_{\alpha}^{\beta}\left(t_{A}\right)_{\gamma}^{\delta}=\frac{C_{2}\left(\mathbf{d}_{\mathrm{TC}}\right) d_{\mathrm{TC}}}{d_{\mathrm{TC}}^{2}-1}\left(\delta_{\alpha}^{\delta} \delta_{\gamma}^{\beta}-\frac{1}{d_{\mathrm{TC}}} \delta_{\alpha}^{\beta} \delta_{\gamma}^{\delta}\right) .
$$

Then, in the large- $N_{\mathrm{TC}}$ limit, $\mathcal{L}_{\mathrm{TC}}$ Fierz-transforms into

$$
\begin{aligned}
\mathcal{L}_{\mathrm{TC}}= & \frac{3 \pi C_{2}\left(\mathbf{d}_{\mathrm{TC}}\right) \alpha_{\mathrm{TC}}(\Lambda)}{d_{\mathrm{TC}} \Lambda^{2}}\left[\bar{T}^{i \alpha} T_{j \alpha} \bar{T}^{j \beta} T_{i \beta}-T^{i \alpha} \gamma_{5} T_{j \alpha} \bar{T}^{j \beta} \gamma_{5} T_{i \beta}\right. \\
& \left.-\frac{1}{2} \bar{T}^{i \alpha} \gamma^{\mu} T_{j \alpha} \bar{T}^{j \beta} \gamma_{\mu} T_{i \beta}-\frac{1}{2} \bar{T}^{i \alpha} \gamma^{\mu} \gamma_{5} T_{j \alpha} \bar{T}^{j \beta} \gamma_{\mu} \gamma_{5} T_{i \beta}\right] .
\end{aligned}
$$

Adding this to the $G_{3}$ term in eq. (2.1), the effective $\lambda$ is

$$
\lambda_{\text {eff }}=\frac{G_{3} d_{\mathrm{TC}} \Lambda^{2}}{8 \pi^{2}}+\frac{3 C_{2}\left(\mathbf{d}_{\mathrm{TC}}\right) \alpha_{\mathrm{TC}}(\Lambda)}{4 \pi}=\lambda+\frac{\alpha_{\mathrm{TC}}(\Lambda)}{4 \alpha_{c}} .
$$

For the critical value $\lambda_{\text {eff }}=1, \lambda=1-\alpha_{\mathrm{TC}}(\Lambda) / 4 \alpha_{c}$. This is less than $20 \%$ higher than $\lambda_{\alpha_{\mathrm{TC}}(\Lambda)}$ for $\alpha_{\mathrm{TC}}(\Lambda) / \alpha_{c}<0.5$, which is the range that Takeuchi considered. Thus, our approximation for $\mathcal{L}_{\mathrm{TC}}$ captures well the main effect of adding TC to ETC in the gap equation and spin-zero scattering amplitudes.

We address at this point the following question: is there an additional spontaneous breaking of EW (or any other) symmetry when TC becomes strong and forms the condensate $\langle\bar{D} D\rangle$ ? The answer is no. The EW symmetry is already broken to $\mathrm{U}(1)_{E M}$ by the ETC interaction. Furthermore, there is no appreciable contribution to the EW order parameter $v$ because $D$-condensate gives rise to no Goldstone boson. The chiral current $\bar{D} \gamma_{\mu} \gamma_{5} D$ has a TC-anomalous divergence and explicit breaking of this symmetry is $\mathcal{O}\left(\Lambda_{\mathrm{TC}}\right)$.

\footnotetext{
${ }^{9}$ Although this term is isospin-symmetric, its strength is not sufficient to produce $m_{D} \neq 0$.
} 


\section{Masses of the spin-one technihadrons}

As we stressed at the outset, the challenge for a TC-based composite Higgs model is to explain convincingly why $H$ is much lighter than the lowest-lying spin-one technihadrons. In our model, there is the additional matter that there are two scales, $\Lambda_{\mathrm{TC}}$ and $\Lambda_{\mathrm{ETC}}=\Lambda$. Which of these controls $M_{\rho_{H}}$ ? If it is just $\Lambda$, are these masses of that order or, as for the Higgs, very much lighter? In this section we present an argument suggesting they are at least as heavy as $\Lambda_{\mathrm{TC}}$ and therefore well above the Higgs mass. For this, we assume that $M_{\rho_{H}}, \ldots$ are due entirely to an ETC interaction and find that this results in unphysical or implausible masses for these states.

We start by considering a simplified model with the doublet $T=(U, D)$ as the only fermions. Its $\mathrm{SU}(2)_{L} \otimes \mathrm{U}(1)_{R}$ invariant ETC interaction is

$$
\mathcal{L}_{T}=G_{3} \bar{T}_{L}^{i \alpha} U_{R \alpha} \bar{U}_{R}^{\beta} T_{L i \beta} .
$$

This interaction produces nonzero $m_{U}$, but not $m_{D}$, if

$$
\frac{G_{3} d_{\mathrm{TC}}}{8 \pi^{2}}\left(\Lambda^{2}-m_{U}^{2} \ln \frac{\Lambda^{2}}{m_{U}^{2}}\right)=1 .
$$

While $\mathcal{L}_{T}$ can generate a light Higgs boson and three Goldstone bosons, it has the wrong chiral structure to generate masses for the spin-one technihadrons in the large- $N_{\mathrm{TC}}$ limit. Therefore, we expand it to include terms capable of this. We assume that ETC generates $V V$ and $A A$ contact interactions which add to $\mathcal{L}_{T}$. For simplicity, we can take them to be $V$ - $A$ symmetric and flavor-U(2) invariant without affecting our argument:

$$
\mathcal{L}_{T}=G_{3}\left[\bar{T}_{L}^{i \alpha} U_{R \alpha} \bar{U}_{R}^{\beta} T_{L i \beta}-\frac{1}{4} \delta \sum_{a=0}^{3}\left(\bar{T}^{\alpha} \gamma^{\mu} \tau_{a} T_{\alpha} \bar{T}^{\beta} \gamma_{\mu} \tau_{a} T_{\beta}+\left(\gamma_{\mu} \rightarrow \gamma_{\mu} \gamma_{5}\right)\right)\right],
$$

where $\tau_{a}$ are Pauli matrices acting in the $(U, D)$-flavor space. The parameter $\delta$ allows freedom in the choice of the ETC coupling of the $V V$ and $A A$ terms. We write (with a unit $\rho_{H}$ coupling to the $\mathrm{U}(2)$ current)

$$
\left\langle\Omega\left|\bar{T}^{\alpha} \gamma_{\mu} \frac{\tau_{A}}{2} T_{\alpha}\right| \rho_{B}(p)\right\rangle=\epsilon_{\mu}(p) \delta_{A B},
$$

where $p^{\mu} \epsilon_{\mu}(p)=0$. Then, to leading order in $N_{\mathrm{TC}}$, the technivector masses are given by the poles in the $\rho_{A} \rightarrow \rho_{B}$ amplitude

$$
\begin{aligned}
\mathcal{T}_{A B}(p)= & \epsilon^{\mu *}(p) \epsilon^{\nu}(p)\left(-2 \delta G_{3}\right)\left[g_{\mu \nu} \delta_{A B}-\frac{1}{2} \delta G_{3} d_{\mathrm{TC}} I_{\mu \nu}^{A B}(p)\right. \\
& \left.+\left(-\frac{1}{2} \delta G_{3} d_{\mathrm{TC}}\right)^{2} \sum_{C} I_{\mu \lambda}^{A C}(p) I_{\lambda \nu}^{C B}(p)+\cdots\right] \\
= & \epsilon^{\mu *}(p) \epsilon^{\nu}(p)\left(-2 \delta G_{3}\right)\left[\left(1+\frac{1}{2} \delta G_{3} d_{\mathrm{TC}} I(p)\right)^{-1}\right]_{\mu \nu}^{A B}
\end{aligned}
$$

where

$$
I_{\mu \nu}^{A B}(p)=i \int \frac{d^{4} k}{(2 \pi)^{4}} \operatorname{Tr}\left[\left(\frac{\not k+\not p+M}{(k+p)^{2}-M^{2}}\right) \gamma_{\mu} \tau_{A}\left(\frac{\not k+M}{k^{2}-M^{2}}\right) \gamma_{\nu} \tau_{B}\right]
$$


In this model, with $m_{D}=0$, the fermion mass matrix is

$$
M=\left(\begin{array}{cc}
m_{U} & 0 \\
0 & 0
\end{array}\right) .
$$

The momentum integral (3.6) is cutoff at $\Lambda$, just as the ones in the spin-zero channels were, giving

$$
\begin{aligned}
I_{\mu \nu}^{11,22}(p)= & -\frac{\Lambda^{2}}{4 \pi^{2}} g_{\mu \nu}+\frac{1}{\pi^{2}} \int_{0}^{1} d x\left[\left(p_{\mu} p_{\nu}-p^{2} g_{\mu \nu}\right) x(1-x)+\frac{1}{2} m_{U}^{2} x g_{\mu \nu}\right] \\
& \times \ln \left(\frac{\Lambda^{2}+m_{U}^{2} x-p^{2} x(1-x)}{m_{U}^{2} x-p^{2} x(1-x)}\right) ; \\
I_{\mu \nu}^{33,00}(p)= & -\frac{\Lambda^{2}}{4 \pi^{2}} g_{\mu \nu}+\frac{1}{2 \pi^{2}} \int_{0}^{1} d x\left(p_{\mu} p_{\nu}-p^{2} g_{\mu \nu}\right) x(1-x) \\
& \times\left[\ln \left(\frac{\Lambda^{2}+m_{U}^{2}-p^{2} x(1-x)}{m_{U}^{2}-p^{2} x(1-x)}\right)+\ln \left(\frac{\Lambda^{2}-p^{2} x(1-x)}{-p^{2} x(1-x)}\right)\right] ; \\
I_{\mu \nu}^{30,03}(p)= & \frac{1}{2 \pi^{2}} \int_{0}^{1} d x\left(p_{\mu} p_{\nu}-p^{2} g_{\mu \nu}\right) x(1-x) \\
& \times\left[\ln \left(\frac{\Lambda^{2}+m_{U}^{2}-p^{2} x(1-x)}{m_{U}^{2}-p^{2} x(1-x)}\right)-\ln \left(\frac{\Lambda^{2}-p^{2} x(1-x)}{-p^{2} x(1-x)}\right)\right] .
\end{aligned}
$$

The $p_{\mu} p_{\nu}$ terms in these integrals do not contribute to $\mathcal{T}_{A B}$. Then, in the leading-log approximation, the $\rho_{H}-\omega_{H}$ mixing term is negligible and the poles in $\mathcal{T}_{A B}$ are at

$$
\begin{array}{rlrl}
1-\frac{\delta G_{3} d_{\mathrm{TC}} \Lambda^{2}}{8 \pi^{2}}-\frac{\delta G_{3} d_{\mathrm{TC}}\left(p^{2}-\frac{3}{2} m_{U}^{2}\right)}{12 \pi^{2}} \ln \left(\frac{\Lambda^{2}}{m_{U}^{2}}\right) & =0 & \text { for } A=B=1,2 ; \\
1-\frac{\delta G_{3} d_{\mathrm{TC}} \Lambda^{2}}{8 \pi^{2}}-\frac{\delta G_{3} d_{\mathrm{TC}} p^{2}}{12 \pi^{2}} \ln \left(\frac{\Lambda^{2}}{m_{U}^{2}}\right)=0 & \text { for } A=B=3,0 .
\end{array}
$$

Using the gap eq. (3.2), the poles in $\mathcal{T}_{11,22}$ and $\mathcal{T}_{33,00}$ are at $\bar{p}^{2}$ satisfying

$$
\begin{array}{ll}
\bar{p}^{2} \ln \left(\frac{\Lambda^{2}}{m_{U}^{2}}\right)=\frac{3}{2}\left(\frac{1-\delta}{\delta}\right)\left[\Lambda^{2}-m_{U}^{2} \ln \left(\frac{\Lambda^{2}}{m_{U}^{2}}\right)\right] & \text { for } \rho_{H}^{ \pm} \\
\bar{p}^{2} \ln \left(\frac{\Lambda^{2}}{m_{U}^{2}}\right)=\frac{3}{2 \delta}\left[(1-\delta) \Lambda^{2}-m_{U}^{2} \ln \left(\frac{\Lambda^{2}}{m_{U}^{2}}\right)\right] & \text { for } \rho_{H}^{0}, \omega_{H} .
\end{array}
$$

This is unphysical unless $0<\delta<1$ for $\rho_{H}^{ \pm}$and $0<\delta<1-m_{U}^{2} / \Lambda^{2} \ln \left(\Lambda^{2} / m_{U}^{2}\right)$ for $\rho_{H}^{0}$ and $\omega_{H}$. For $\delta$ at its upper limit, $\bar{p}^{2} \simeq 0$, i.e., very much less than $\Lambda_{\mathrm{TC}}^{2}$. We believe this is unreasonable because no symmetry is responsible for such light masses. Our calculations break down beyond the $\Lambda$-cutoff, so $M_{\rho_{H}} \gtrsim \Lambda$ is an unreliable result. Over a large part of the physical range of $\delta, \Lambda_{\mathrm{TC}}^{2} \ll \bar{p}^{2} \simeq \Lambda^{2} / \ln \left(\Lambda^{2} / m_{U}^{2}\right)<\Lambda^{2}$. We cannot exclude this. But, for a mass we have assumed is generated solely by strong ETC, it seems implausible to us. A more believable result is that TC generates the $\rho_{H}$ and $\omega_{H}$ masses and that they are of order $\Lambda_{\mathrm{TC}}$, the scale at which $\alpha_{\mathrm{TC}}$ becomes large and TC interactions confine. In both the latter two cases, the $\rho_{H}, \omega_{H}$ masses are significantly larger than the Higgs mass, and that is a necessary condition for the viability of this type of model. 
Let us extend this argument to the full $G_{1}-G_{2}-G_{3}$ model. There are two obvious possibilities for the $V V+A A$ terms: we could add just the $\delta G_{3}$ interaction as we did in eq. (3.3) or we could add similar terms with the appropriate coefficient, $-\frac{1}{4} \delta G_{i}$, to all three interactions. The gap-equation condition is now given by eq. (1.7). In the first case, the poles are always at $\bar{p}^{2} \sim \Lambda^{2} / \ln \left(\Lambda^{2} / m_{U}^{2}\right)$, which we believe is implausible. The second case is similar to the pure- $G_{3}$ model discussed above. Finally, similar results and conclusions hold for ETC-generated masses of the axial vectors $a_{H}$; they are either unreasonably small or much larger than $\Lambda_{\mathrm{TC}}$ but smaller than $\Lambda$. The conclusion we draw is that an ETC origin of the technivector masses is less plausible than that they arise from the confined $\mathrm{TC}$ interactions and are of $\mathcal{O}(1 \mathrm{TeV})$.

\section{Phenomenology of $\rho_{H}$ and $a_{H}$}

Preliminary remarks about the phenomenology of the model's technifermion bound states were made in ref. [16]. They included, in particular, the expectations that: (1) the most accessible low-lying states, in addition to the Higgs $H$ and longitudinal weak bosons $W_{L}$ and $Z_{L}$ (which really are bound by the ETC interaction, eq. (1.2)), are the spin-one, techniisospin one and zero $\rho$ and $\omega$-like composites; (2) their masses are $\sim 1 / 2-2 \mathrm{TeV}$ and they are produced at the LHC via the Drell-Yan process; (3) their principal decay modes would be to $W_{L}^{+} W_{L}^{-}, W_{L}^{ \pm}, Z_{L}$ or $W_{L}^{+} W_{L}^{-} Z_{L}$ and to $W_{L} H, Z_{L} H$. In this section we refine - and correct - these expectations, presenting some specific predictions of production and decay rates. We concentrate on the $I=1$ vectors and axial vectors, $\rho_{H}$ and $a_{H}$, which have simple two-body decay modes and Drell-Yan-size production rates.

The technicolor interaction governing $\rho_{H}$ and $a_{H}$ is invariant under parity and techniisospin of $(U, D)$. This symmetry is broken by the electroweak gauge interaction and the $U-D$ mass difference. The first is $\mathcal{O}(\alpha)$ and the second is an $I=1$ operator and does not contribute to mass splitting within the isotriplet multiplets. Furthermore, because the ETC interaction, eq. (1.2), is tuned to be close to the EW phase transition, we expect that $\rho_{H}$ and $a_{H}$ are nearly parity-doubled triplets with $M_{\rho_{H}} \cong M_{a_{H}}$. Thus, they can be adequately described as the gauge bosons of a hidden local symmetry (HLS) [49], $\mathrm{SU}(2)_{L} \otimes \mathrm{SU}(2)_{R}$, with equal gauge couplings $g_{L}=g_{R} \equiv g_{\rho_{H}}$ [50-53]. This coupling is analogous to $g_{\rho \pi \pi}$ and is expected to be large, $g_{\rho_{H}} \simeq 3-5$. The equality $g_{L}=g_{R}$ makes the $\rho_{H}-a_{H}$ contribution to the $S$-parameter [54-58] small [50,51]. The dimension-three and four interactions of $\rho_{H}, a_{H}$ with EW gauge bosons and the Higgs respect parity-invariance up to EW corrections.

The principal $\rho_{H}, a_{H}$ decay modes are to lighter states with $\bar{T} T$ content, namely, the longitudinally-polarized $V_{L}=W_{L}, Z_{L}$ and the Higgs boson $H .{ }^{10}$ The two-body decays allowed by parity and isospin are

$$
\begin{array}{ll}
\rho_{H}^{0} \rightarrow W_{L}^{+} W_{L}^{-}, & \rho_{H}^{ \pm} \rightarrow W_{L}^{ \pm} Z_{L} ; \\
a_{H}^{0} \rightarrow Z_{L} H, & a_{H}^{ \pm} \rightarrow W_{L}^{ \pm} H .
\end{array}
$$

\footnotetext{
${ }^{10}$ If $\rho_{H}$ is coupled to third-generation quarks by an ETC interaction of strength $\mathcal{O}\left(G_{2}\right)$, eqs. (1.6), (1.7) imply the resulting decay rate to $\bar{t} t$ or $\bar{b} t$ is suppressed by the tiny factor $\left(M_{\rho_{H}} / \Lambda\right)^{4}$. This is much smaller than the small $\mathcal{O}\left(g^{4} / g_{\rho_{H}}^{2}\right) \bar{t} t$-rate induced by mixing with the EW bosons.
} 
There is no allowed $\rho_{H}^{0} \rightarrow Z_{L} Z_{L}$. The fact that $V_{L}, H$ also contain third generation quarks may deplete somewhat the $\rho_{H}, a_{H}$ couplings to them. This does not alter the major decay modes in eqs. (4.1), (4.2) nor affect their production rates at the LHC. In the absence of significant depletion, the relevant $\rho_{H}, a_{H}$ couplings are induced by their $\mathcal{O}\left(g M_{\rho_{H}}^{2} / g_{\rho_{H}}\right)$ mixing with the EW gauge bosons $[25,51]$. They are

$$
\begin{aligned}
\mathcal{L}\left(\rho_{H} \rightarrow V V\right)= & -\frac{i g^{2} g_{\rho_{H}} v^{2}}{2 M_{\rho_{H}}^{2}} \rho_{H \mu \nu}^{0} W_{\mu}^{+} W_{\nu}^{-}-\frac{i g^{2} g_{\rho_{H}} v^{2}}{2 M_{\rho_{H}}^{2} \cos \theta_{W}}\left(\rho_{H \mu \nu}^{+} W_{\mu}^{-}-\rho_{H \mu \nu}^{-} W_{\mu}^{+}\right) Z_{\nu} ; \\
\mathcal{L}\left(a_{H} \rightarrow V H\right)= & g g_{\rho_{H}} v\left(a_{H \mu}^{+} W_{\mu}^{-}+a_{H \mu}^{-} W_{\mu}^{+}\right) H+\frac{g g_{\rho_{H}} v}{\cos \theta_{W}} a_{H \mu}^{0} Z_{\mu} H ; \\
\mathcal{L}\left(a_{H} \rightarrow V V\right)= & \frac{i g^{2} g_{\rho_{H}} v^{2}}{2 M_{\rho_{H}}^{2}} a_{H \mu}^{0}\left(W_{\mu \nu}^{+} W_{\nu}^{-}-W_{\mu \nu}^{-} W_{\nu}^{+}\right) \\
& -\frac{i g^{2} g_{\rho_{H}} v^{2}}{2 M_{\rho_{H}}^{2} \cos \theta_{W}}\left[a_{H \mu}^{+}\left(W_{\nu}^{-} Z_{\mu \nu}-W_{\mu \nu}^{-} Z_{\nu}\right)-\text { h.c. }\right],
\end{aligned}
$$

where $G_{\mu \nu}=\partial_{\mu} G_{\nu}-\partial_{\nu} G_{\mu}, g$ is the weak-SU(2) coupling, and $v=246 \mathrm{GeV}$. For $M_{\rho_{H}, a_{H}} \gg M_{V, H}$, the decay rates implied by these interactions overwhelmingly involve $V_{L}$ and are $\mathcal{O}\left(g^{0}\right)$ :

$$
\begin{gathered}
\Gamma\left(\rho_{H}^{0} \rightarrow W^{+} W^{-}\right) \cong \Gamma\left(\rho_{H}^{ \pm} \rightarrow W^{ \pm} Z\right) \cong \frac{g_{\rho_{H}}^{2} M_{\rho_{H}}}{48 \pi} ; \\
\Gamma\left(a^{0} \rightarrow Z H\right) \cong \Gamma\left(a^{ \pm} \rightarrow W^{ \pm} H\right) \cong \frac{g_{\rho_{H}}^{2} M_{a_{H}}}{48 \pi} ; \\
\Gamma\left(a_{H}^{0} \rightarrow W^{+} W^{-}\right) \cong \Gamma\left(a_{H}^{ \pm} \rightarrow W^{ \pm} Z\right) \cong \frac{g_{\rho_{H}}^{2} M_{W}^{2} M_{a_{H}}^{3}}{24 \pi M_{\rho_{H}}^{4}} .
\end{gathered}
$$

The decay rates $\Gamma\left(\rho_{H} \rightarrow V V\right)$ and $\Gamma\left(a_{H} \rightarrow V H\right)$ are nearly identical because, as explained above, $M_{\rho_{H}} \cong M_{a_{H}}$ and $\left(H, \boldsymbol{V}_{L}\right)$ are an approximately degenerate $(2,2)$ quartet in the Wigner-Weyl mode of the symmetry [53]. In eq. (4.8), $a_{H} \rightarrow V_{L} V_{T}$, hence the $M_{W}^{2} / M_{\rho_{H}}^{2}=\mathcal{O}\left(g^{2} / g_{\rho_{H}}^{2}\right)$ suppression of that rate. The decay rate of $\rho_{H}^{0} \rightarrow Z_{L} Z_{T}$ is similarly suppressed.

The $\rho_{H}$ and $a_{H}$ are produced at the LHC mainly by the Drell-Yan (DY) mechanism of $\bar{q} q$ annihilation. The $\rho_{H}$ and $a_{H}$ have only very weak direct coupling to light quarks, induced by ETC. Thus, their DY production also proceeds through their mixing with the electroweak bosons. ${ }^{11}$ A secondary source of $\rho_{H}$ production is weak-vector boson fusion (VBF). This VBF is dominated by $V_{L} V_{L}$ fusion and, so, it is negligibly small for $a_{H}$ production in our model. This will be important in distinguishing the nearly degenerate $\rho_{H}$ and $a_{H}$ from each other.

CMS [29-31] and ATLAS [26-28] reported studies of highly-boosted $V V$ and $V H$ pairs in their Run 1 data at $8 \mathrm{TeV}$. Both collaborations observed resonance-like excesses of $2-3 \sigma$ at $1.8-2.0 \mathrm{TeV}$, near the upper end of the mass range at which we would expect to find $\rho_{H} \rightarrow V V$ and $a_{H} \rightarrow V H$. These excesses were discussed and their significances and production rates estimated from the Run 1 data in ref. [59].

\footnotetext{
${ }^{11} \mathrm{By}$ a slight abuse of language, we shall refer to the HLS gauge bosons and the corresponding masseigenstates as $\rho_{H}$ and $a_{H}$.
} 


\begin{tabular}{|c|c|c|c|}
\hline$M_{\rho_{H}}(\mathrm{GeV})$ & $\Gamma\left(\rho_{H} \rightarrow V V\right)(\mathrm{GeV})$ & $\Gamma\left(a_{H} \rightarrow V H\right)(\mathrm{GeV})$ & $\Gamma\left(a_{H} \rightarrow V V\right)(\mathrm{GeV})$ \\
\hline 1800 & 178 & 184 & 0.82 \\
1900 & 188 & 196 & 0.78 \\
2000 & 198 & 208 & 0.74 \\
\hline
\end{tabular}

Table 2. Principal decay rates of the isovector bosons $\rho_{H}$ and $a_{H}$ for $g_{\rho_{H}}=3.862$ and $M_{a_{H}}=$ $1.05 M_{\rho_{H}}$; from ref. [25]. These widths may be reduced by a factor of two or so by the $\bar{t} t$ and $\bar{b} t$ content of $H, W_{L}, Z_{L}$. Because the diboson decay modes are still dominant, this does not affect their production rates.

\begin{tabular}{|c|c|c|c|c|c|}
\hline$\sqrt{s}$ & $M_{\rho_{H}}(\mathrm{GeV})$ & $\sigma\left(\rho_{H}^{ \pm}\right)_{D Y+V B F}(\mathrm{fb})$ & $\sigma\left(\rho_{H}^{0}\right)_{D Y+V B F}(\mathrm{fb})$ & $\sigma\left(a_{H}^{ \pm}\right)(\mathrm{fb})$ & $\sigma\left(a_{H}^{0}\right)(\mathrm{fb})$ \\
\hline 8 & 1800 & $1.53+0.36$ & $0.74+0.18$ & 0.71 & 0.37 \\
8 & 1900 & $1.05+0.24$ & $0.50+0.12$ & 0.51 & 0.27 \\
8 & 2000 & $0.73+0.15$ & $0.36+0.075$ & 0.36 & 0.17 \\
\hline 13 & 1800 & $7.61+3.67$ & $3.74+1.93$ & 4.65 & 2.23 \\
13 & 1900 & $5.74+2.62$ & $2.81+1.37$ & 3.16 & 1.69 \\
13 & 2000 & $4.37+1.90$ & $2.16+0.99$ & 2.39 & 1.27 \\
\hline
\end{tabular}

Table 3. Production cross sections at the LHC of the isovector bosons $\rho_{H}$ and $a_{H}$ for $g_{\rho_{H}}=3.862$ and $M_{a_{H}}=1.05 M_{\rho_{H}}\left(\rho_{H}^{ \pm}=\rho_{H}^{+}+\rho_{H}^{-}\right)$. The individual DY $+\mathrm{VBF}$ contributions are given for $\rho_{H}$; the VBF rates for $a_{H}$ are very small and not given. For $g_{\rho_{H}}=2.73, \sigma\left(\rho_{H} \rightarrow V V\right)$ is $50 \%$ larger, $\sigma\left(a_{H} \rightarrow V H\right)$ is doubled, and their widths are half as large as in table 2. No $K$-factor has been applied to the cross sections; from ref. [25].

We proposed in ref. [25] that these excesses are due to production of the $\rho_{H}$ and $a_{H}$ modes in eqs. (4.1), (4.2). The decay rates and cross sections for $M_{\rho_{H}}=1.8-2.0 \mathrm{TeV}$, $M_{a_{H}}=1.05 M_{\rho_{H}}$ and $g_{\rho_{H}}=1.9 \mathrm{TeV} / 2 v=3.862$ (i.e., $\left.M_{\rho_{H}} \simeq \frac{1}{2} g_{\rho_{H}}(4 v)\right)$ are given in tables 2 and $3 .^{12}$ The diboson resonance cross sections in table 3 for $\sqrt{s}=8 \mathrm{TeV}$ are $\sim 2-10$ times smaller than those estimated in ref. [59] from ATLAS and CMS Run 1 data; see figure 3. On the other hand, they are typical of what would be expected for Drell-Yan rates for $\simeq 2 \mathrm{TeV} \rho$-like and $W^{\prime} / Z^{\prime}$ bosons decaying to dibosons. We shall have more to say on this below.

The cross sections at $\sqrt{s}=13 \mathrm{TeV}$ are $5-7$ times larger than at $8 \mathrm{TeV}$. Preliminary reports of 2.6-3.2 $\mathrm{fb}^{-1}$ of $13-\mathrm{TeV}$ data by CMS [62] and ATLAS [63-65] neither confirmed nor excluded the Run 1 excesses. In August 2016, the LHC collaborations reported searches for the heavy diboson resonances using $13-15 \mathrm{fb}^{-1}$ of data taken at $13 \mathrm{TeV}$. In the diboson mass range 1.8-2.0 TeV, the ATLAS 95\% CL upper limit on cross section times branching ratio was $\gtrsim 20 \mathrm{fb}$ in the all-hadronic $(q q q q)$ channels [66] as well as in the semileptonic chan-

\footnotetext{
${ }^{12}$ The Drell-Yan rates in table 3 were calculated using the couplings of ref. [60], appropriate to a single fermion doublet, for which we assume electric charges $\pm \frac{1}{2}$. The DY cross sections given in ref. [60] are easily modified for the case at hand in which there are no other light PGBs. They are encoded in PyTHIA 6.4 [61]. Cross sections for $\left(Q_{U}, Q_{D}\right)=(0,-1)$ differ only slightly from these and give no contribution to the $H \rightarrow \gamma \gamma$ rate via a $U$-loop.
} 


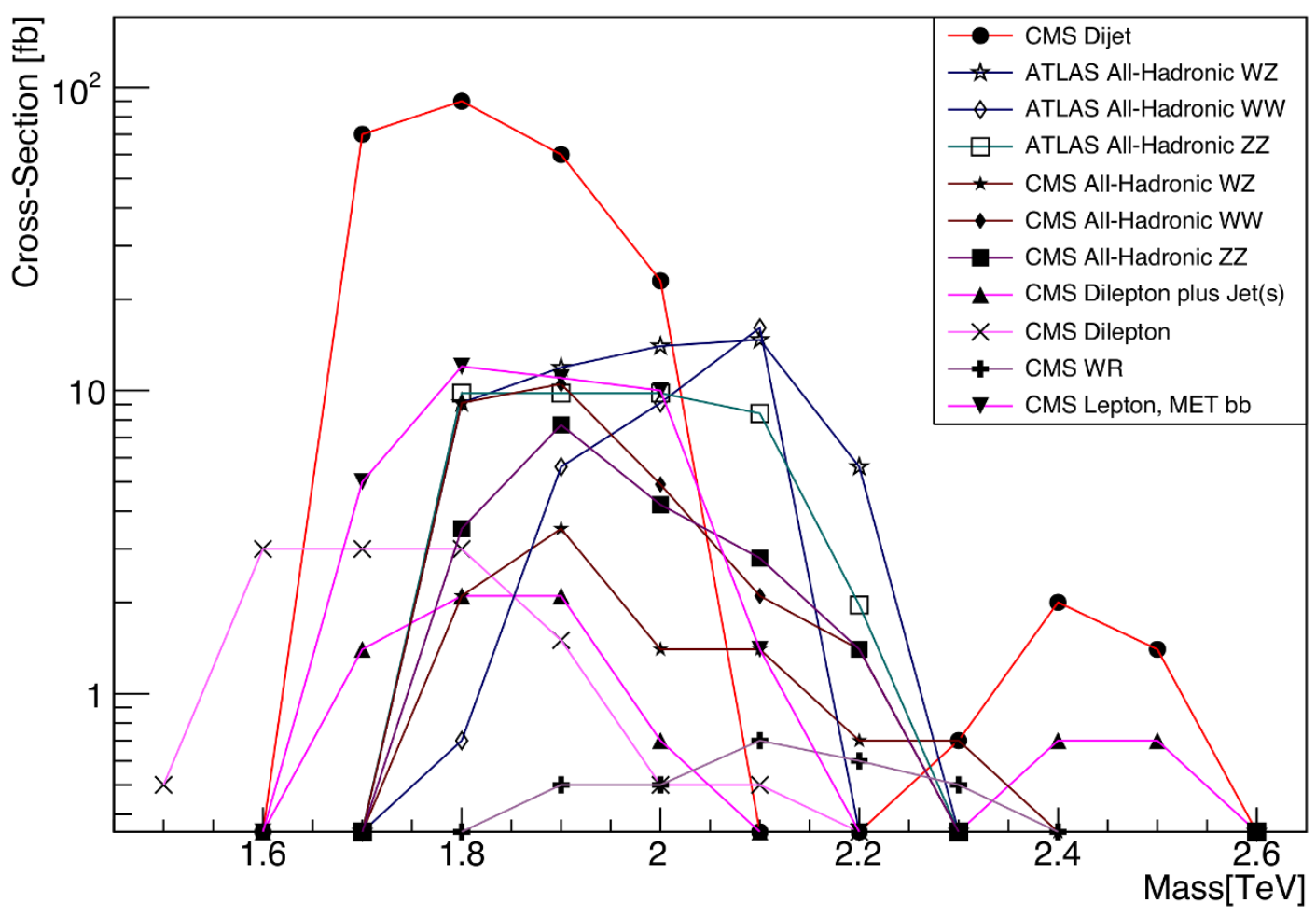

Figure 3. Cross sections for ATLAS and CMS Run 1 diboson excesses estimated from significances greater than $1.5 \sigma$; from ref. [59]. The CMS dijet with an estimated cross section of $\sim 100 \mathrm{fb}^{-1}$ was not seen in Run 2 data.

nels $\ell \nu q q$ for $\ell=\mu, e$ [67]. Its upper limit was 5-8 fb in the sum of the semileptonic channels $\ell^{+} \ell^{-} q q$ and $\nu \nu q q$ (dominated, of course, by $\nu \nu q q$ ) [68]. The early Run 2 data from CMS $\left(12.9 \mathrm{fb}^{-1}\right)$ put a $95 \%$ CL limit on the $\ell \nu q q$ channel that was also $20 \mathrm{fb}$ [69]. The ATLAS search for a $W / Z+H$ resonance in the $q q b b$ channel yielded a limit of $\sim 12 \mathrm{fb}^{-1}$ [67]. Results based on the current Run 2 data sets of $36 \mathrm{fb}^{-1}$ are appearing. In the all-hadronic modes $W W, W Z \rightarrow q q q q$ CMS reported 95\% CL limits of $10 \mathrm{fb}$ for $Z^{\prime} \rightarrow W W$ and $W^{\prime} \rightarrow W Z$ at $M_{W^{\prime}, Z^{\prime}} \simeq 2 \mathrm{TeV}$ [70] and 3-4fb for $W^{\prime} \rightarrow W H \rightarrow q q b b$ and $Z^{\prime} \rightarrow Z H \rightarrow q q b b$, also at $M_{W^{\prime}, Z^{\prime}} \simeq 2 \mathrm{TeV}$ [71]. ATLAS has reported a search for $Z^{\prime} \rightarrow Z H$ and $W^{\prime} \rightarrow W H$ in these hadronic modes. Its $95 \% \mathrm{CL}$ limits at $M_{W^{\prime}, Z^{\prime}}=2 \mathrm{TeV}$ are $3 \mathrm{fb}$ and $6 \mathrm{fb}$, respectively [72]. Taking into account $W, Z$ leptonic branching ratios where appropriate, all these limits are above, but beginning to close in on our predictions in table 3 . In any case, it is clear that the diboson excesses of Run 1 - especially those in the all-hadronic channels depicted in figure 3 - were, at best, large up-fluctuations.

Our proposal leads to several observations, predictions and recommendations for Runs $2+3$ data analyses:

1) Given the rather small rates in table 3 and the low efficiency of separating $W$ and $Z$ in their hadronic decay modes, greater sensitivity to resonance signals may be had in the early Run 2 data by combining them, i.e., lump together the presumed $W W$, $W Z$ and $Z Z$ all-hadronic data, the $\ell \nu W$ and $\ell \nu Z$ data, etc. 
2) The only $V V$ diboson resonances come from $\rho_{H}$ production. Isospin invariance implies equal decay rates to $W^{ \pm} Z$ and $W^{+} W^{-}$, but no $Z Z$-signal. ${ }^{13}$ It is therefore desirable that the separation of $p_{T} \sim 1 \mathrm{TeV}$, hadronically-decaying $W$ and $Z$-bosons be sharpened, and the overlap between all-hadronic $W W, W Z$ and $Z Z$ selections be minimized. Until that is possible, semileptonic and all-leptonic $V V$ events will be needed determine the content of the diboson resonances. This may be feasible in Run 3 with its planned luminosity of $300 \mathrm{fb}^{-1}$.

3) The $V H$ resonances in our model are due to $a_{H}$, not $\rho_{H}$, production, but they are expected to be nearly degenerate with the $V V$ resonances. The $\rho_{H}$ may be distinguished by looking for forward jets. At $\sqrt{s}=13 \mathrm{TeV}$, about $1 / 3$ of $\rho_{H} \rightarrow V V$ production is due to VBF, which is accompanied by forward jets with a rapidity gap. The $a_{H} \rightarrow V H$ process is due entirely to DY because $a_{H} \rightarrow V V$ is so strongly suppressed and, so, it has no forward jets.

4) Table 3 shows that $\sigma\left(\rho_{H}^{ \pm} \rightarrow W^{ \pm} Z\right): \sigma\left(\rho_{H}^{0} \rightarrow W^{+} W^{-}\right) \simeq \sigma\left(a_{H}^{ \pm} \rightarrow W^{ \pm} H\right): \sigma\left(a_{H}^{0} \rightarrow\right.$ $Z H) \simeq 2$. This is a consequence of the approximate parity-doubling and the proton's parton luminosities at high mass. Another consequence of the parton luminosities is that $\sigma\left(\rho_{H}^{+}, a_{H}^{+}\right) \simeq 2 \times \sigma\left(\rho_{H}^{-}, a_{H}^{-}\right)$.)

5) The large widths of $\rho_{H}$ and $a_{H}$ reflect their underlying strong dynamics, i.e., $g_{\rho_{H}} \simeq 3-5$, as well as to their decays to $V_{L}$. Heavy $W^{\prime}$ and $Z^{\prime}$ bosons are also expected to decay to $W_{L}^{ \pm} Z_{L}$ and $W_{L}^{+} W_{L}^{-}$, but to be relatively narrow because their gauge couplings and mixings to $W, Z$ are weak. In either case, greater sensitivity may be obtained by detection methods favoring longitudinal polarization. It seems that, at least for now, the best path to diboson width measurements is through semileptonic $V$-decays. Again, we expect the same widths for $V V$ and $V H$ resonances.

6) Our model is distinguished from ones in which the composite Higgs is a pseudoGoldstone boson in two ways. First, if $H$ is a PGB, there generally are top and $W$-partners that keep it light. They are not hadrons of the strong dynamics that bind $H$ and, so, are lighter than the $2-\mathrm{TeV} \rho_{H}$ and $a_{H}$. They should show up soon at the LHC. On the other hand, there are no top and $W$-partners needed in the strongETC model, and there aren't any. Second, the PGB models predict corrections to the $H$ couplings with EW bosons and fermions that may be observable at the LHC [59]. Such corrections in our model are suppressed by $\left(M_{W} / M_{\rho_{H}}\right)^{2}$, too small to be detected at the LHC.

\section{$5 \quad$ Summary and plans}

In this paper we developed further our strong-ETC model of electroweak symmetry breaking and updated our discussion of the diboson resonances $\rho_{H}$ and $a_{H}$ and their standing vis-à-vis the latest LHC data. We stressed that weak-TC - meaning a minimal role for

\footnotetext{
${ }^{13}$ If a $Z Z$ signal is confirmed, it must be due to production of another state, e.g., an analog of the $f_{0}(980)$.
} 
TC in EWSB - is a necessary ingredient of our model if it is to explain the large mass gap between the Higgs boson $H(125)$ and technihadrons. Our two main theoretical purposes were to include the effect of weak TC on EWSB and to establish as well as we could that the model provides a plausible explanation for the lightness of $H$ relative to the technihadrons $\rho_{H}$ and $a_{H}$. For the first, we used Takeuchi's analysis [24] to show that weak TC modifies only slightly the analysis of ref. [16] in which TC was ignored altogether. Specifically, in the $J^{P}=0^{ \pm}$channels of fermion-fermion scattering, the effective TC interaction has the same form and sign as the corresponding ETC interaction, thus having the effect of requiring only a slightly smaller ETC coupling to trigger EWSB with a light Higgs and three Goldstone bosons $\left(H, \boldsymbol{V}_{L}\right)$.

Our argument that $M_{\rho_{H}} \gg M_{H}$ was an indirect one: we showed that an assumed ETC interaction designed to generate a $\rho_{H}$ pole in technifermion scattering amplitudes leads to values of $M_{\rho_{H}}$ which are either nearly zero or else much greater than $\Lambda_{\mathrm{TC}}$ but less than $\Lambda_{\mathrm{ETC}}$. We have not found an argument more direct than this. We regard both possibilities as implausible compared to $M_{\rho_{H}, a_{H}}=\mathcal{O}\left(\Lambda_{\mathrm{TC}}\right)$ - the scale of their binding interaction - and significantly greater than $M_{H}$. Neither did we address the question of why $\Lambda_{\mathrm{TC}}=\mathcal{O}(1 \mathrm{TeV})$ if $\mathrm{TC}$ has little to do with EWSB. Of course, we expect that it is if the diboson excesses near $2 \mathrm{TeV}$ turn out to be confirmed in LHC Runs $2+3$. But that's not an answer. If the dibosons are not confirmed, the question is moot. That would be unfortunate because our model does not appear to have another readily accessible "smoking-gun" prediction.

On the phenomenological side, in section 4 we reviewed our expectations for the $\rho_{H}$ and $a_{H}$ widths and production cross sections [25], compared these with the latest data from Run 2, and suggested and refined ways to search for them and distinguish our model for the diboson resonances from others that have been proposed (reviewed in ref. [59]). If they are confirmed, there will be plenty for the experimentalists to do to reveal their nature and the interactions responsible for them.

For our model, the main task remaining is to carry out a renormalization group analysis for the Higgs and heavy fermion masses. If this analysis can produce results in accord with experiment, particularly $M_{H}$ below $m_{t}$, it will give strong support to our approach to understanding the Higgs as a light composite state.

\section{Acknowledgments}

We have benefited from conversations with T. Appelquist, K. Black, T. Bose, G. Brooijmans, R. Contino, E. Eichten, B. Holdom, A. Martin, A. Pomerol, S. Rappoccio, T. Takeuchi, J. Thaler and B. Zhou. KL gratefully acknowledges support from several sources during the course of this work: the Labex ENIGMASS (CNRS) during 2014-16 and the Laboratoire d'Annecy-le-Vieux de Physique Théorique (LAPTh) for its continuing hospitality; the CERN Theory Group for support and hospitality in 2014-16 and CERN for a Scientific Associateship in 2015. This research was supported in part by the U.S. Department of Energy under Grant No. DE-SC0010106. 
Open Access. This article is distributed under the terms of the Creative Commons Attribution License (CC-BY 4.0), which permits any use, distribution and reproduction in any medium, provided the original author(s) and source are credited.

\section{References}

[1] ATLAS collaboration, Observation of a new particle in the search for the Standard Model Higgs boson with the ATLAS detector at the LHC, Phys. Lett. B 716 (2012) 1 [arXiv: 1207.7214] [INSPIRE].

[2] CMS collaboration, Observation of a new boson at a mass of $125 \mathrm{GeV}$ with the CMS experiment at the LHC, Phys. Lett. B 716 (2012) 30 [arXiv:1207.7235] [INSPIRE].

[3] S. Weinberg, Implications of Dynamical Symmetry Breaking: An Addendum, Phys. Rev. D 19 (1979) 1277 [INSPIRE].

[4] L. Susskind, Dynamics of Spontaneous Symmetry Breaking in the Weinberg-Salam Theory, Phys. Rev. D 20 (1979) 2619 [INSPIRE].

[5] R. Foadi, M.T. Frandsen and F. Sannino, $125 \mathrm{GeV}$ Higgs boson from a not so light technicolor scalar, Phys. Rev. D 87 (2013) 095001 [arXiv:1211.1083] [INSPIRE].

[6] A. Belyaev, M.S. Brown, R. Foadi and M.T. Frandsen, The Technicolor Higgs in the Light of LHC Data, Phys. Rev. D 90 (2014) 035012 [arXiv: 1309.2097] [InSPIRE].

[7] K. Yamawaki, M. Bando and K.-i. Matumoto, Scale Invariant Technicolor Model and a Technidilaton, Phys. Rev. Lett. 56 (1986) 1335 [INSPIRE].

[8] W.D. Goldberger, B. Grinstein and W. Skiba, Distinguishing the Higgs boson from the dilaton at the Large Hadron Collider, Phys. Rev. Lett. 100 (2008) 111802 [arXiv:0708.1463] [INSPIRE].

[9] T. Appelquist and Y. Bai, A Light Dilaton in Walking Gauge Theories, Phys. Rev. D 82 (2010) 071701 [arXiv: 1006.4375] [INSPIRE].

[10] B. Bellazzini, C. Csáki, J. Hubisz, J. Serra and J. Terning, A Higgslike Dilaton, Eur. Phys. J. C 73 (2013) 2333 [arXiv:1209.3299] [INSPIRE].

[11] R. Contino, The Higgs as a Composite Nambu-Goldstone Boson, arXiv:1005.4269 [INSPIRE].

[12] G.F. Giudice, Naturalness after LHC8, PoS(EPS-HEP 2013) 163 [arXiv: 1307.7879] [INSPIRE].

[13] B. Bellazzini, C. Csáki and J. Serra, Composite Higgses, Eur. Phys. J. C 74 (2014) 2766 [arXiv: 1401.2457] [INSPIRE].

[14] J. Barnard, T. Gherghetta, T.S. Ray and A. Spray, The Unnatural Composite Higgs, JHEP 01 (2015) 067 [arXiv: 1409.7391] [INSPIRE].

[15] Particle Data Group collaboration, K.A. Olive et al., Review of Particle Physics, Chin. Phys. C 38 (2014) 090001 [INSPIRE].

[16] K. Lane, A composite Higgs model with minimal fine-tuning: The large- $N$ and weak-technicolor limit, Phys. Rev. D 90 (2014) 095025 [arXiv:1407.2270] [INSPIRE].

[17] T. Appelquist, M. Einhorn, T. Takeuchi and L.C.R. Wijewardhana, Higher Mass Scales and Mass Hierarchies, Phys. Lett. B 220 (1989) 223 [INSPIRE]. 
[18] R.S. Chivukula, A.G. Cohen and K.D. Lane, Aspects of Dynamical Electroweak Symmetry Breaking, Nucl. Phys. B 343 (1990) 554 [InSPIRE].

[19] W.A. Bardeen, C.T. Hill and M. Lindner, Minimal Dynamical Symmetry Breaking of the Standard Model, Phys. Rev. D 41 (1990) 1647 [InSPIRE].

[20] G. Panico and A. Wulzer, The Composite Nambu-Goldstone Higgs, Lect. Notes Phys. 913 (2016) pp.1-316 [arXiv:1506.01961] [INSPIRE].

[21] T.P. Cheng, E. Eichten and L.-F. Li, Higgs Phenomena in Asymptotically Free Gauge Theories, Phys. Rev. D 9 (1974) 2259 [InSPIRE].

[22] E. Eichten and K.D. Lane, Dynamical Breaking of Weak Interaction Symmetries, Phys. Lett. 90B (1980) 125 [INSPIRE].

[23] T. Appelquist, M. Soldate, T. Takeuchi and L. Wijewardhana, Effective four fermion interactions and chiral symmetry breaking, World Scientific Pub Co. (1988).

[24] T. Takeuchi, Analytical and Numerical Study of the Schwinger-dyson Equation With Four Fermion Coupling, Phys. Rev. D 40 (1989) 2697 [InSPIRE].

[25] K. Lane and L. Pritchett, Heavy Vector Partners of the Light Composite Higgs, Phys. Lett. B 753 (2016) 211 [arXiv: 1507.07102] [InSPIRE].

[26] ATLAS collaboration, Search for high-mass diboson resonances with boson-tagged jets in proton-proton collisions at $\sqrt{s}=8 \mathrm{TeV}$ with the ATLAS detector, JHEP 12 (2015) 055 [arXiv: 1506.00962] [INSPIRE].

[27] ATLAS collaboration, Search for production of $W W / W Z$ resonances decaying to a lepton, neutrino and jets in pp collisions at $\sqrt{s}=8 \mathrm{TeV}$ with the ATLAS detector, Eur. Phys. J. C 75 (2015) 209 [arXiv: 1503.04677] [inSPIRE].

[28] ATLAS collaboration, Search for a new resonance decaying to a $W$ or $Z$ boson and a Higgs boson in the $\ell \ell / \ell \nu / \nu \nu+b \bar{b}$ final states with the ATLAS detector, Eur. Phys. J. C 75 (2015) 263 [arXiv: 1503.08089] [INSPIRE].

[29] CMS collaboration, Search for massive resonances decaying into pairs of boosted bosons in semi-leptonic final states at $\sqrt{s}=8 \mathrm{TeV}$, JHEP 08 (2014) 174 [arXiv:1405.3447] [INSPIRE].

[30] CMS collaboration, Search for massive resonances in dijet systems containing jets tagged as $W$ or $Z$ boson decays in pp collisions at $\sqrt{s}=8$ TeV, JHEP 08 (2014) 173 [arXiv: 1405.1994] [INSPIRE].

[31] CMS collaboration, Search for massive WH resonances decaying into the $\ell \nu \mathrm{b} \overline{\mathrm{b}}$ final state at $\sqrt{s}=8 \mathrm{TeV}$, Eur. Phys. J. C 76 (2016) 237 [arXiv:1601.06431] [InSPIRE].

[32] Y. Nambu and G. Jona-Lasinio, Dynamical Model of Elementary Particles Based on an Analogy with Superconductivity. I, Phys. Rev. 122 (1961) 345 [INSPIRE].

[33] Y. Nambu and G. Jona-Lasinio, Dynamical model of elementary particles based on an analogy with superconductivity. II, Phys. Rev. 124 (1961) 246 [INSPIRE].

[34] Y. Nambu, Bootstrap symmetry breaking in electroweak unification, EFI-89-08 [INSPIRE].

[35] V.A. Miransky, M. Tanabashi and K. Yamawaki, Dynamical Electroweak Symmetry Breaking with Large Anomalous Dimension and t Quark Condensate, Phys. Lett. B 221 (1989) 177 [INSPIRE].

[36] V.A. Miransky, M. Tanabashi and K. Yamawaki, Is the $t$ Quark Responsible for the Mass of $W$ and $Z$ Bosons?, Mod. Phys. Lett. A 4 (1989) 1043 [INSPIRE]. 
[37] B.A. Dobrescu and C.T. Hill, Electroweak symmetry breaking via top condensation seesaw, Phys. Rev. Lett. 81 (1998) 2634 [hep-ph/9712319] [INSPIRE].

[38] R.S. Chivukula, B.A. Dobrescu, H. Georgi and C.T. Hill, Top Quark Seesaw Theory of Electroweak Symmetry Breaking, Phys. Rev. D 59 (1999) 075003 [hep-ph/9809470] [INSPIRE].

[39] H.S. Fukano and K. Tuominen, A hybrid $4^{\text {th }}$ generation: Technicolor with top-seesaw, Phys. Rev. D 85 (2012) 095025 [arXiv: 1202.6296] [INSPIRE].

[40] H.S. Fukano and K. Tuominen, $126 \mathrm{GeV}$ Higgs boson in the top-seesaw model, JHEP 09 (2013) 021 [arXiv: 1306.0205] [INSPIRE].

[41] S. Bar-Shalom, Dynamical Origin for the $125 \mathrm{GeV}$ Higgs; a Hybrid setup, PoS (EPS-HEP 2013) 258 [arXiv: 1310.2942] [INSPIRE].

[42] M. Geller, S. Bar-Shalom and A. Soni, Hybrid dynamical electroweak symmetry breaking with heavy quarks and the $125 \mathrm{GeV}$ Higgs boson, Phys. Rev. D 89 (2014) 035012 [arXiv: 1302.2915] [INSPIRE].

[43] S. Di Chiara, R. Foadi and K. Tuominen, $125 \mathrm{GeV}$ Higgs from a chiral techniquark model, Phys. Rev. D 90 (2014) 115016 [arXiv:1405.7154] [INSPIRE].

[44] S. Di Chiara, R. Foadi, K. Tuominen and S. Tähtinen, Dynamical Origin of the Electroweak Scale and the $125 \mathrm{GeV}$ Scalar, Nucl. Phys. B 900 (2015) 295 [arXiv:1412.7835] [inSPIRE].

[45] G. von Gersdorff, E. Pontón and R. Rosenfeld, The Dynamical Composite Higgs, JHEP 06 (2015) 119 [arXiv: 1502.07340] [INSPIRE].

[46] A.G. Cohen and H. Georgi, Walking Beyond the Rainbow, Nucl. Phys. B 314 (1989) 7 [INSPIRE].

[47] K.D. Lane, Asymptotic Freedom and Goldstone Realization of Chiral Symmetry, Phys. Rev. D 10 (1974) 2605 [INSPIRE].

[48] K.D. Lane and M.V. Ramana, Walking technicolor signatures at hadron colliders, Phys. Rev. D 44 (1991) 2678 [INSPIRE].

[49] M. Bando, T. Kugo and K. Yamawaki, Nonlinear Realization and Hidden Local Symmetries, Phys. Rept. 164 (1988) 217 [INSPIRE].

[50] R. Casalbuoni, A. Deandrea, S. De Curtis, D. Dominici, R. Gatto and M. Grazzini, Degenerate BESS model: The possibility of a low-energy strong electroweak sector, Phys. Rev. D 53 (1996) 5201 [hep-ph/9510431] [INSPIRE].

[51] K. Lane and A. Martin, An Effective Lagrangian for Low-Scale Technicolor, Phys. Rev. D 80 (2009) 115001 [arXiv:0907.3737] [INSPIRE].

[52] B. Bellazzini, C. Csáki, J. Hubisz, J. Serra and J. Terning, Composite Higgs Sketch, JHEP 11 (2012) 003 [arXiv: 1205.4032] [INSPIRE].

[53] T. Appelquist, Y. Bai, J. Ingoldby and M. Piai, Spectrum-doubled Heavy Vector Bosons at the LHC, JHEP 01 (2016) 109 [arXiv:1511.05473] [INSPIRE].

[54] D.C. Kennedy and B.W. Lynn, Electroweak Radiative Corrections with an Effective Lagrangian: Four Fermion Processes, Nucl. Phys. B 322 (1989) 1 [InSPIRE].

[55] M.E. Peskin and T. Takeuchi, A new constraint on a strongly interacting Higgs sector, Phys. Rev. Lett. 65 (1990) 964 [INSPIRE]. 
[56] M. Golden and L. Randall, Radiative Corrections to Electroweak Parameters in Technicolor Theories, Nucl. Phys. B 361 (1991) 3 [InSPIRE].

[57] B. Holdom and J. Terning, Large corrections to electroweak parameters in technicolor theories, Phys. Lett. B 247 (1990) 88 [INSPIRE].

[58] G. Altarelli, R. Barbieri and S. Jadach, Toward a model independent analysis of electroweak data, Nucl. Phys. B 369 (1992) 3 [inSPIRE].

[59] J. Brehmer et al., The Diboson Excess: Experimental Situation and Classification of Explanations; A Les Houches Pre-Proceeding, arXiv:1512.04357 [INSPIRE].

[60] K. Lane and S. Mrenna, The Collider phenomenology of technihadrons in the technicolor straw man model, Phys. Rev. D 67 (2003) 115011 [hep-ph/0210299] [INSPIRE].

[61] T. Sjöstrand, S. Mrenna and P.Z. Skands, PYTHIA 6.4 Physics and Manual, JHEP 05 (2006) 026 [hep-ph/0603175] [inSPIRE].

[62] CMS collaboration, Search for massive resonances decaying into pairs of boosted $W$ and $Z$ bosons at $\sqrt{s}=13 \mathrm{TeV}$, CMS-PAS-EXO-15-002.

[63] ATLAS collaboration, Search for resonances with boson-tagged jets in $3.2 \mathrm{fb}^{-1}$ of $p$ p collisions at $\sqrt{s}=13 \mathrm{TeV}$ collected with the ATLAS detector, ATLAS-CONF-2015-073.

[64] ATLAS collaboration, Search for new resonances decaying to a $W$ or $Z$ boson and a Higgs boson in the $\ell^{+} \ell^{-} b \bar{b}, \ell \nu b \bar{b}$ and $\nu \bar{\nu} b \bar{b}$ channels with pp collisions at $\sqrt{s}=13 \mathrm{TeV}$ with the ATLAS detector, Phys. Lett. B 765 (2017) 32 [arXiv:1607.05621] [INSPIRE].

[65] ATLAS collaboration, Search for $W W / W Z$ resonance production in the $\ell \nu q q$ final state at $\sqrt{s}=13 \mathrm{TeV}$ with the ATLAS detector at the LHC, ATLAS-CONF-2015-075.

[66] ATLAS collaboration, Search for resonances with boson-tagged jets in $15.5 \mathrm{fb}^{-1}$ of $\mathrm{pp}$ collisions at $\sqrt{s}=13 \mathrm{TeV}$ collected with the ATLAS detector, ATLAS-CONF-2016-055.

[67] ATLAS collaboration, Search for diboson resonance production in the $\ell \nu q q$ final state using pp collisions at $\sqrt{s}=13 \mathrm{TeV}$ with the ATLAS detector at the LHC, ATLAS-CONF-2016-062.

[68] ATLAS collaboration, Searches for heavy $Z Z$ and $Z W$ resonances in the llqq and $\nu \nu q q$ final states in pp collisions at $\sqrt{s}=13 \mathrm{TeV}$ with the ATLAS detector, ATLAS-CONF-2016-082.

[69] CMS collaboration, Search for new resonances decaying to $\mathrm{WW} / \mathrm{WZ} \rightarrow \ell \nu \mathrm{qq}$, CMS-PAS-B2G-16-020.

[70] CMS collaboration, Search for massive resonances decaying into $W W, W Z, Z Z, q W$ and $q Z$ in the dijet final state at $\sqrt{s}=13 \mathrm{TeV}$, CMS-PAS-B2G-17-001.

[71] CMS collaboration, Search for heavy resonances decaying into a vector boson and a Higgs boson in hadronic final states with 2016 data, CMS-PAS-B2G-17-002.

[72] ATLAS collaboration, Search for Heavy Resonances Decaying to a $W$ or $Z$ Boson and $a$ Higgs Boson in the $q \bar{q}^{(\prime)} b \bar{b}$ Final State in pp Collisions at $\sqrt{s}=13$ TeV with the ATLAS Detector, ATLAS-CONF-2017-018. 